\title{
¿Cuándo deben intervenir los entrenadores en la dirección de un partido de voleibol? Posibles intervenciones psicológicas durante las distintas fases competitivas de un partido
}

\author{
When must coaches intervene in the direction of Volleyball match? \\ Possible psychological interventions during the \\ different competitive phases of a match
}

\section{Quando que os treinadores devem interferir na direçáo de um jogo de voleibol? Possíveis intervençóes psicológicas durante as distintas fases competitivas de um jogo de voleibol}

\author{
Joaquín Díaz Rodríguez* y Isabel Díaz Ceballos** \\ ${ }^{*}$ Comité Nacional de Entrenadores de Voleibol. Federación Española de Voleibol. Asociación Psicología del Deporte APD-Cantabria. \\ ** Universidad de Cantabria.
}

Resumen: El objetivo de este trabajo es aportar sugerencias de actuación psicológica que permitan a los entrenadores a ser mas eficaces en el trabajo de dirección de equipo durante la competición en concreto, y principalmente durante un partido. Se repasan las características de las distintas fases y periodos competitivos que ocurren durante los encuentros de voleibol y se sugieren cuales son las intervenciones psicológicas del entrenador mas apropiadas en cada una de esas fases orientadas al mantenimiento o mejora de la disposición mental de los jugadores.

Palabras clave: Intervención, entrenadores, competición, voleibol

Abstract: The objective of this paper is to bring suggestions for psychological proceedings that will enable coaches to become more efficient in the handling of the teams during competitions, more precisely and mainly during the game. The features of the different phases and periods of volleyball competitions are analyzed and there are some suggestions about what psychological interventions are more appropriate for a coach to use in each of the phases. Such interventions are oriented toward the maintenance and improvement of the mental disposition of the players.

Key words: Interventions / coaches / competition / volleyball

Resumo: O objetivo desse trabalho é de proporcionar sugestôes de atuação psicológica que permitam a eficácia do trabalho dos treinadores na organização e direção de sua equipe durante uma competição e principalmente durante um jogo.

Recordamos as distintas características das fases e períodos competitivos do voleibol e sugerimos as intervençóes psicológicas apropriadas do treinador em cada uma das fases, sempre orientando a melhora da disposição mental dos jogadores.

Palavras chave: Intervenção, treinadores, voleibol.

\section{Introducción}

La importancia de la función del entrenador tanto en los deportes individuales como colectivos es reconocida por todos los estamentos implicados en la actividad deportiva. Todos reconocen que el entrenador es una de las figuras clave tanto en el proceso de enseñanza deportiva como en el rendimiento deportivo. Esta importancia ha sido recogida por numero-

Correspondencia. Joaquín Díaz Rodríguez, APD-Cantabria C/ Mártires, 16 60 Izda. 39300 Torrelavega Cantabria. Espańa. E-mail quinichi@ hotmail.com. sísimos autores y en diferentes deportes colectivos, muchos de los cuales proceden del campo de la psicología del deporte. (Cruz, 1994; Alonso, Boixados y Cruz; 2007; Balaguer, 2007; Buceta, 2004; Giménez, 2003; Romero, 2008; Cantón, 2006) La actuación del entrenador durante la competición deportiva de voleibol también ha sido referida por diferentes autores procedentes del mundo del entrenamiento deportivo. (Díaz, 1992; Herrera, Ramos y Mirella, 1989; Santos, 1992; Ureńa, 1992; Cunha, 1998; Moreno, Santos, Ramos, Sanz, Fuentes, del Villar, 2002; Mesquita, Gisela, Rosado, Moreno, 


\section{5; Velasco 2009).}

Las circunstancias reglamentarias ofrecen nuevas posibilidades de dirección del equipo durante los partidos a los entrenadores.

En este trabajo trataremos de concretar las características de las diferentes fases de un encuentro de voleibol. Haremos especial referencia a las peculiaridades de los subperiodos puramente competitivos. Aportaremos algunas sugerencias y actuaciones y cuales serán las conductas mas apropiadas del entrenador en cada una de esas fases.

\section{Fases durante un encuentro de voleibol}

El voleibol es un deporte en el que se suceden las acciones de forma continuada, sistemática y cíclica. Presenta un alto grado de incertidumbre en la que es difícil preveer la duración de los sets y los partidos y los tipos de situaciones que pueden ocurrir (Ureńa, 1992)... Una de las exigencias mentales del voleibol es la rapidez con la que se suceden las distintas jugadas y la participación colectiva e individual en cada una de ellas. Otra de las características psicológicas del partido es la aparición de diferentes exigencias mentales para los jugadores en una misma fase dependiendo de los tanteos o resultados parciales. También los sucesivos cambios de complejos tácticos, que la mayoría de autores (Santos, 1992) resumen en dos: complejo uno (K-I) y complejo dos (K-II).

En el complejo K-II se encuentra aquel equipo que está en posesión de saque y su actitud debe ser prioritariamente defensiva. En el complejo K-I se encuentra el equipo que debe recibir el saque y su actitud prioritaria debe ser ofensiva. Los encuentros son una sucesión alternada de cambios de un complejo a otro.

Sirva este esquema básico para entender la dinámica del juego. En síntesis el juego consiste en devolver el balón al equipo contrario de tal forma que le sea muy difícil de controlar.

El juego y la reglamentación permiten espacios temporales para planificar y actuar con cierta anticipación. Como muestra Díaz (1992) en la competición y el partido el éxito en múltiples ocasiones depende de las decisiones que tome el entrenador durante los momentos competitivos. Los entrenadores deben actuar con diligencia en cada uno de los periodos competitivos tratando de controlar y mejorar tanto las acciones individuales como colectivas para lograr el objetivo común o de equipo.

El momento cumbre de todo hecho deportivo en la competición es el partido. Todo el trabajo del entrenador tiene como objetivo que el equipo llegue al partido en las mejores condiciones para realizar durante juego todas las estrategias y acciones que hemos preparado. La actuación del entrenador, por tanto, durante el partido es fundamental para conseguir el mayor rendimiento. Durante el partido, el entrenador, en- tre otras cosas, debe tratar de dirigir a sus jugadores, aplicar medidas, tomar decisiones, aportar soluciones, motivar, controlar emociones, comunicar ordenes y modificar realidades. En este trabajo trataremos de contribuir con el trabajo de los entrenadores aportándoles informaciones sobre las características de las fases de un partido en las que serán más efectivas sus intervenciones. Las características de las fases en las que los jugadores podrán sacar mayor provecho de su dirección. Cuales serán las fases competitivas más idóneas para la intervención técnico-táctica o psicológica. Cuales serán los mejores momentos para intervenir para controlar el nivel de activación, fijar mejor su foco atención o incidir en la mejor colaboración de los jugadores.

Siguiendo la propuesta de Buceta (2004) para el baloncesto pero que se utiliza en todos los deportes colectivos, dividiremos el periodo competitivo de un partido de voleibol de en las siguientes fases:

- FASE PREVIA

- FASE COMPETITIVA

- FASE POSTCOMPETITIVA

Para nuestro propósito en este trabajo subdividiremos la fase competitiva en tres subperiodos:

- Periodo de participación activa intensa

- Periodos de pausa

- Periodos de participación activa de intensidad menor.

Por lo que ampliaríamos las tres fases competitivas clásicas como se observa en el siguiente cuadro

Cuadro 1.Esquema de los periodos competitivos en voleibol

FASE PREVIA

FASE COMPETITIVA

Periodo de participación de actividad intensa

Periodos de participación activa de intensidad menor

Periodos de pausa

FASE POSTCOMPETITIVA

\section{Fase previa}

Nosotros consideramos la fase previa como el tiempo que transcurre entre la entrada de los jugadores a la cancha para comenzar su calentamiento hasta el inicio del partido. Es el momento inmediatamente anterior a comenzar a competir. Según las distintas competiciones el tiempo es variable. No obstante, podemos ampliar esa fase previa a un tiempo más amplio. De hecho algunas de las sugerencias de intervención que aquí proponemos requieren de un mayor tiempo.

El objetivo de la intervención psicológica del entrenador en la fase previa se dirige, prioritariamente, a que el jugador consiga un estado físico, psicológico y de relación grupal optimo. Son momentos de una gran importancia en la prepa- 
ración del partido ya que son los últimos para poder dar las últimas consignas antes del comienzo del partido.

\section{Los jugadores en la fase previa}

En la fase previa, por lo general, pero dependiendo de la trascendencia de la competición y del partido, los jugadores suelen tener un nivel de activación alto. Presentan cierta inestabilidad emocional y posiblemente excesiva motivación inicial ya que la competición en si misma es muy motivante.

La fase previa, por tanto, no es el momento más adecuado para inundar a los jugadores con cantidad de información, ya que su situación mental no es la más adecuada para aprovechar las indicaciones o propuestas del entrenador.

\section{Los entrenadores en la fase previa}

En general, y según los trabajos de Moreno, Santos y Del Villar (2005), los entrenadores aportan información principalmente colectiva (63\%) e individual (37\%) que se reparte entre el colocador principal del equipo (44\%) y atacantes (18\%). La información verbal que se refiere al equipo contrario es el $(55 \%)$ y $(40 \%)$ al equipo propio. Añaden estos mismos autores que incluyen otras informaciones que consideran relevantes: árbitro, luz, balones, humedad...etc. Mucha de la información que los entrenadores tienden a trasmitir en la fase previa a un partido, es aconsejable que la realicen en las vísperas del partido (noche anterior o jornada de mañana si el partido es por la tarde).

Los entrenadores en sus actuaciones en esta fase previa deben tener en cuenta la situación psicológica en la que están sus jugadores y recordar la finalidad que se quieren conseguir en esta fase previa. Como hemos comentado anteriormente, se trata de que el jugador consiga su mejor estado general para competir. Creemos que el entrenador debe ser muy selectivo en la información. La información que aporte debe de ser breve y concisa. Debe conseguir que los jugadores se responsabilicen de su propio acondicionamiento general para la competición. En este acondicionamiento no debe olvidar los aspectos mentales y grupales. . Moreno, Santos y Del Villar (2005) insisten en la importancia de la actuación de los entrenadores para transmitir la información adecuada durante la fase previa.

\section{Intervenciones de los entrenadores en la fase previa}

En la fase previa, como sugieren distintos autores (Buceta 2004; Díaz, 1992), los entrenadores en sus intervenciones deben vigilar la comunicación no verbal más que la información verbal. Los jugadores, en esos momentos previos, suelen atender más a los gestos que a las palabras. Las comunicaciones e informaciones deben ser concretas y cortas. Deben centrarse más en actitudes y comportamientos que en el recordatorio de actuaciones y situaciones técnico-táctico. Es recomendable poner el acento más en nuestras fortalezas que en las fortalezas del rival.

Coincidimos con Díaz (1992) en que los entrenadores deben permanecer atentos a lo que está haciendo cada jugador, llamando la atención a aquellos que aparentan falta de concentración en su trabajo. La conducta y actitud del entrenador debe transmitir confianza y tranquilidad.

Según nuestra experiencia creemos que algunas de las intervenciones que los entrenadores realizan en esta fase, deben ser realizadas en otros momentos tanto competitivos como, principalmente, pre-competitivos. Las intervenciones en esta fase deben ser muy cortas y positivas. Son mas recomendadas las intervenciones individuales y de grupos pequeños.

Cuadro 2. Intervenciones de los entrenadores en la fase previa

Transmitir confianza en las posibilidades del equipo y de los jugadores

Controlar las expectativas aparentes

Pedir comunicación y compromiso

Anticipar situaciones estresantes

Controlar nivel de activación adecuado.

Cuadro 3. Sugerencias de actuación de los entrenadores durante la fase previa

Decir a los jugadores qué se espera de ellos.

Preparar las dificultades. Se han debido entrenar anteriormente

Información breve de conductas prioritarias de cada uno.

Dar pocas instrucciones: precisas, claras, breves y acordes con la situación

Transmitir tranquilidad tanto en la comunicación verbal como no verbal

El entrenador podrá dirigirse a algún jugador que ha manifestado en alguna ocasión algunas expectativas aparentes. p.e. aquellos jugadores que manifiestan que va a ser "un partido fácil" o aquellos otros que manifiestan "un partido imposible de ganar"

Deberá dirigirse brevemente a aquel jugador que observe sobre activado o híper motivado, y tratar de que regule su activación.

El contacto físico, el comentario positivo, el refuerzo social, la sonrisa y la tranquilidad son comportamientos adecuados y necesarios en las fases previas.

El entrenador deberá evitar comentarios del tipo;" hay que ganar este partido" "hay que salir a muerte" por su inconcreción y falta de propuesta adecuada de actuación.

Insistimos que las intervenciones mas adecuadas son las individuales, ya que el jugador en esta fase previa, como hemos comentado, tendrá un alto grado de motivación y un 
nivel de activación grande y le será más fácil prestar atención a las sugerencias e indicaciones del entrenador de manera personal. Las actuaciones para el grupo completo deberán ser las últimas. El entrenador deberá controlar ese tiempo y llamar a sus jugadores con tiempo suficiente antes de las indicaciones finales.

\section{Fase competiva}

La fase competitiva es la competición misma, el partido. En el transcurso de un partido de voleibol distinguiremos diferentes periodos de actividad y de participación de los jugadores. Diferentes momentos de intensidad que van a depender de las diversas circunstancias del juego.

Como ya hemos comentado, el voleibol es un deporte cíclico y secuenciado con una sucesión continuada de complejos tácticos de ataques y defensas con una participación desigual de jugadores. Mentalmente el funcionamiento de los jugadores suele ser diferente tanto en su participación en la jugada o secuencia de juego como en las posibilidades para recibir información. En algunas fases de juego los jugadores disponen de un mayor tiempo para analizar la situación y realizar las acciones, para comunicarse con los compañeros y adoptar alternativas o recibir información. Sin embargo en otros momentos esas actuaciones son imposibles de realizar.

Siguiendo nuestra propuesta dividiremos la fase competitiva, según sea la participación de los jugadores en la misma y la actividad que realiza, en tres subperiodos: Periodos de participación activa intensa, periodos de pausa y periodos de participación activa de intensidad menor

\section{Periodos de participación activa intensa.}

Los periodos de participación activa intensa se caracterizan porque el jugador participa en la jugada de forma directa e intensa. Es el protagonista la jugada, el actor del gesto técnico o bien participa junto con el actor protagonista.

Según Susan y Mihaly, (2002) el jugador en estos momentos de participación activa intensa se encuentra "en un estado de conciencia en el que el jugador llega a estar totalmente absorbido por lo que está haciendo excluyendo todo tipo de pensamiento o emoción" Veamos algunos ejemplos:

El jugador en el momento que ejecuta se encuentra en un momento de participación activa intensa. Otros momentos de participación activa intensa son: el colocador y el rematador de la jugada. Los jugadores que realizan el apoyo a su rematador. El jugador que recibe el saque. El bloqueador o bloqueadores. Los defensores Todos estos jugadores son los protagonistas principales de la jugada. Aunque la mayor parte de los jugadores participan de manera activa el protagonismo de unos y otros no es el mismo. Sin embargo todos los jugadores se encuentran totalmente absorbidos por la acción deportiva. Son momentos de intensa actividad física y técnica

Intervenciones psicológicas en las fases de participación activa intensa. Dado que en estos momentos los jugadores están muy concentrados lo mas acertado es "dejarlos jugar". Coincidimos con Hotz (1999) en que en estos momentos en los que los jugadores realizan sus acciones técnicas no están en condiciones de llevar a la práctica las indicaciones del entrenador. Por supuesto que las indicaciones que se hacen en ese mismo instante no son percibidas por el protagonista de la jugada.

Según Buceta (2004), si el entrenador intenta darles instrucciones durante estos periodos, es muy probable que estas interfieran en la atención que el jugador necesita para rendir en su juego. Por lo tanto, dado el nivel de conciencia y atención que presentan los jugadores en estos momentos de participación activa intensa no es recomendable la intervención de los entrenadores.

Hemos observado que hay entrenadores que intervienen durante estas fases de juego instigando al jugador para que realice o corrija alguna acción técnica (p. e; les indican que remate en una dirección concreta o que tapen una zona especifica de bloqueo, en el preciso momento que el jugador la está ejecutando). Como venimos comentando, estas actuaciones del entrenador son, además de inapropiadas, ineficaces e inútiles. No obstante, hay algunas intervenciones del entrenador que pueden ser útiles tratando de incitar a algún jugador durante estos momentos de participación activa intensa. Según Buceta (2004) con jugadores jóvenes, en periodos de formación puede ser útil para favorecer determinadas conductas. Para ello el entrenador debe haber entrenado estas órdenes en las sesiones de entrenamiento. Los jugadores deben identificar las órdenes que el entrenador quiere trasmitir. El entrenador debe utilizar una palabra enérgica y corta para dar una orden concreta: "duro","finta" "fuera". En el voleibol esta condición se puede producir en aquellos jugadores que no son los protagonistas absolutos de la jugada. p.e. El jugador que debe apoyar el remate, o la corrección de una posición defensiva.

\section{Sugerencias de actuaciones de los entrenadores en los periodos de participación activa intensa}

En estos periodos de intensidad en el juego, los entrenadores deben mantenerse serenos y controlados. Estas habilidades psicológicas de tranquilidad y de control emocional son fundamentales en el entrenamiento personal de los entrenadores. Los entrenadores tendrán que trasmitir adecuadamente estas habilidades para que los jugadores no las interpreten como no estar involucrados en la dinámica de juego. Para aquellos entrenadores que no tengan implantadas estas habilidades psicológicas sería muy recomendable que recibieran entrenamiento en técnicas de control emocional. Un adecuado en- 
trenamiento psicológico y formación de los entrenadores les permitirá durante estos momentos de fases de participación activa intensa incrementar y mejorar su actuación, por ejemplo para atender adecuadamente, al desarrollo y evolución del partido, lo que se suele llamar; "leer adecuadamente el encuentro", es decir; interpretar correctamente todos los estímulos relevantes tanto técnicos como tácticos que ocurren en la cancha. También para transmitir adecuadamente las emociones y vivencias del partido.

Cuando el entrenador es consciente de estas conductas podrá adoptar y tomar las decisiones mas adecuadas, Por ejemplo; podría intentar convertir periodos de gran presión en un periodo más pausado, utilizando, en estos casos, a aquellos jugadores más idóneos (cambios) o intentar convertir periodos de gran presión en periodos mas pausados (cambiar ritmos de partido, utilizar los tiempos muertos, agotar tiempos de saque...)

El refuerzo de la conducta o del gesto técnico realizado por sus jugadores son otras de las sugerencias de actuación de los entrenadores durante los periodos de participación activa intensa, justo inmediatamente después de terminar una jugada. Los comentarios del entrenador y los gestos de aprobación son conductas especialmente interesantes, sobre todo en los jugadores jóvenes. Frases del entrenador como: "muy bien", "eso es", "así" "fenómeno" cuando haya finalizado una acción o un gesto técnico exitoso, ayudarán a consolidar esas conductas y hacer que el jugador las introduzca en su repertorio técnico-táctico. De la misma forma el entrenador no deberá afear o reforzar de forma negativa aquel gesto técnico no exitoso o mal realizado. La conducta mas apropiada del entrenador en estos periodos de máxima activación sobre los errores será ignorarlos para intervenir y actuar en otros momentos.

Los entrenadores durante estos periodos deberán prestar un máximo cuidado, cuando los "sets" o los partidos llegan a sus puntos finales en los que la intensidad del juego y la activación de los jugadores aumentan. También deberá prestar una máxima vigilancia cuando los marcadores son muy apretados o cuando el tanteo nos es adverso. Es en estos momentos cuando los entrenadores psicológicamente orientados y entrenados muestran su mayor control para conseguir mejores actuaciones.

Ya hemos advertido que en una fase de participación activa intensa no todos lo jugadores tienen el mismo protagonismo en toda la serie de la jugada. Por ejemplo, en el complejo $\mathrm{KI}$; en la fase de recepción el protagonista es el jugador que realiza la recepción, el resto de los jugadores no son los protagonistas. En la fase de ataque el protagonista principal es el rematador y el resto de los jugadores son activos. En el complejo KII los bloqueadores son los protagonistas, el resto de los jugadores son muy activos pero no protagonizan principalmente la acción. Por tanto las intervenciones del entrenador pueden ser distintas para los jugadores y dependiendo del complejo táctico en el que se encuentren. En equipos y jugadores poco experimentados y en deportistas jóvenes suelen ocurrir situaciones de "despiste" y perdida de concentración. Los entrenadores y los propios jugadores podrían intervenir para focalizar la atención y controlar esos despistes y ausencias. Por ejemplo no acudir a los apoyos del rematador o tener una posición demasiado alta en defensa. La intervención del entrenador, en estos casos, puede resultar muy recomendable. Por supuesto que estas intervenciones deben ser individuales y personalizadas.

\section{Actuación y participación de los jugadores en los periodos de participación activa intensa}

Como hemos comentado hay jugadores menos activos en estos periodos intensos. Son los jugadores que no son los protagonistas principales de la jugada. Para estos jugadores es recomendable que los entrenadores les orienten e incluso les pongan tareas que les ayuden a mantener un adecuado nivel de activación. Las tareas psicológicas estarán relacionadas con ejercicios de focalización de la atención sobre estímulos discriminatorios que les posibiliten abordar jugadas posteriores similares. Por ejemplo, el entrenador puede sugerir zonas y alturas del remate. Llamar la atención sobre la calidad de la recepción del equipo rival. Advertir sobre los jugadores fintadotes. Informar sobre la versatilidad de saque del equipo contrario.

En algunas ocasiones, después de estos trabajos de atención sobre los jugadores que no protagonizan la jugada, éstos suelen tomar decisiones e iniciativas propias que benefician al grupo. Por ejemplo en los apoyos o anticipaciones imprevistas.

Una intervención por parte de los entrenadores de carácter especial durante estos periodos de intensa actividad será sobre los jugadores que no están en cancha. Estos jugadores no participan pero deben mantener un comportamiento adecuado con el momento que se está jugando. El entrenador les debe asesorar sobre que deben permanecer tranquilos, pero activos e involucrados. Les debe insistir en que se mantengan preparados y conscientes de que pueden ser requeridos para participar en cualquier momento en estos periodos del partido.

\section{Participación activa de intensidad menor.}

Los periodos de participación activa de intensidad menor son aquellos en los que se produce una pausa en la intensidad. Son los que aparecen en el siguiente cuadro.

Cuadro 4. Periodos de participación activa de intensidad menor 


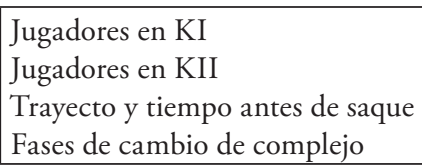

La principal característica de estos subperíodos competitivos, de gran importancia en el transcurso del partido, es que en ellos se produce una pausa en la intensidad de las demandas físicas y técnicas y un aumento de las demandas psicológicas y tácticas. Estos subperiodos son momentos de espera. Como manifiesta Dosil (2002): en los periodos de espera hay un tiempo para pensar, Durante este tiempo los jugadores pueden adelantar acontecimientos, analizar jugadas, pensar en fallos anteriores y preocuparse por acontecimientos y cuestiones ajenas al juego. Son, por tanto, momentos de gran trabajo mental y de gran importancia en el trabajo psicológico de los entrenadores

Se encuentran en estos periodos los jugadores que en el complejo KI están esperando el saque para su recepción. Los jugadores que en el complejo KII se encuentran a la espera de que su jugador realice el saque. Los jugadores que están en la zona de red prestos para realizar el bloqueo El jugador que efectúa el saque en el trayecto y en los segundos antes de efectuarle. Todas estas situaciones son las que incluimos en los subperiodos de participación activa de intensidad menor. Es precisamente a estos momentos a los que se refiere Beal (1989) cuando afirma que hay que utilizar estos momentos para orientar a los jugadores hacia aspectos tácticos o Velasco (2009) cuando incide que estos son momentos para corregir errores repetidos por los jugadores.

Recordar que el tiempo reglamentario que el jugador tiene para sacar es de ocho segundos. Aunque, como recuerda el trabajo de Díaz y Rodríguez (2005,) la mayoría de los jugadores no agotan el tiempo reglamentario para efectuar su saque, si se puede llegar a aumentar hasta agotar el tiempo con un adecuado entrenamiento y esta puede ser un magnifico tiempo para la intervención de los entrenadores sobre los jugadores en estos momentos de intensidad activa menor.

Los tiempos de participación activa de intensidad menor son muy numerosos y continuos. Por ejemplo, en un set con un resultado de 25-20 se han tenido 45 momentos de estos subperiodos. En el supuesto de que cada equipo hubiera gastado los 8 segundos reglamentarios, situación que como hemos comentado es infrecuente, habríamos sumado la cantidad de 6 minutos en ese hipotético resultado parcial. Por tanto, el tiempo de esos subperiodos en los que los jugadores se encuentran en una actividad menor, y por tanto receptivos a las informaciones e intervenciones de los entrenadores, es muy superior a los tiempos de pausa solicitados por el entrenador y los tiempos técnicos reglamentarios.

\section{Actuaciones de los entrenadores en los periodos}

de participación activa de intensidad menor.

Estos periodos, por tanto, son muy apropiados para que los entrenadores intervengan, informan y asesoren a sus jugadores. Los entrenadores disponen de tiempo para observar a su equipo y al equipo contrario. Pueden utilizar esos segundos para introducir algunas correcciones tácticas. Ofrecer información relevante a sus jugadores y dar información del equipo contrario. También el entrenador del equipo que se encuentra en el complejo KII puede aprovechar este tiempo para mejorar la posición de sus receptores. En el otro caso el entrenador que su equipo se encuentre en el complejo KI puede asesorar sobre la zona de saque a su jugador en saque o indicar las posibles zonas de bloqueo preferente. Son periodos de gran intensidad y de enorme posibilidad de actuaciones por parte de entrenadores y jugadores.

En este sentido debemos destacar el trabajo de Cloes, Delhaes y Pieron (1993) citado por Moreno, Santos y Villar (2005) en el que enumera que las intervenciones del entrenador durante el partido pertenecen a la categoría de "incitación a la acción” para motivar, animar, y elogiar o apoyar psicológicamente. Nosotros creemos que en el voleibol esos momentos son los periodos de participación activa de intensidad menor. Este momento de "incitación a la acción" es el periodo de participación activa de intensidad menor.

Efectivamente, coincidimos con los trabajos de Moreno, Santos, Ramos, Sanz y Fuentes (2002) en que estos momentos son muy adecuados para que el entrenador realice un mayor porcentaje de acciones psicológicas, pero también son momentos muy adecuados para intervenir sobre acciones tácticas tanto individuales como colectivas

Hay una característica que vamos a destacar de estos periodos y que condiciona la calidad de la intervención de los entrenadores, así como sobre la necesidad del entrenamiento psicológico del propio entrenador.

Las intervenciones del entrenador pueden estar, y de hecho nuestra experiencia nos demuestra, muy condicionadas por el resultado de la acción de la jugada que acaba de finalizar. Es decir; según que el resultado de la jugada sea positivo o negativo así será la comunicación tanto verbal como no verbal que realiza el entrenador. El resultado de la jugada condicionará el contenido y la forma de la información que va a trasmitir. Por lo general, cuando el equipo ha conseguido un resultado positivo el entrenador tiende a reforzar positivamente con palmadas, apoyo psicológico y elogios de carácter colectivo. Cuando el resultado no es positivo disminuyen los refuerzos verbales y no verbales y las informaciones e intervenciones suelen ser de tipo técnico-táctico. Estas circunstancias deben ser analizadas y trabajadas. El entrenador, mejorará sus intervenciones durante estos periodos si atiende y trabaja su formación psicológica personal. La actuación de un psicólogo del deporte trabajando directamente con el entrenador opti- 
mizará sus actuaciones.

Insistiremos una vez más, en la necesidad de que el entrenador posea un alto grado de control emocional. Entre otras muchas posibilidades le permitirá una adecuada actuación en la comunicación verbal y no verbal.

Así mismo, un adecuado control emocional le permitirá interpretar mejor los rendimientos durante la competición lo que provocará mejores intervenciones. En general el entrenamiento en control emocional de los entrenadores les permitirá ser constantes en sus intervenciones tanto en los éxitos como en los fracasos.

En todas las modalidades deportivas los entrenadores están sometidos a una gran presión mental. Es en los en los puntos finales de los partidos y en los resultados parciales y los marcadores s ajustados donde mas se sufre esa presión. Es en estos momentos que generalmente van seguidos de un periodo de actividad de intensidad menor, en los que los entrenadores se van a beneficiar de un adecuado entrenamiento mental que le ayudará en sus funciones de dirección de equipo.

En los cuadros siguientes ofrecemos algunas actuaciones de los entrenadores en los periodos de participación activa de intensidad menor. En ellos se recogen actuaciones del entrenador. Trabajos para el entrenamiento mental del propio entrenador y actuaciones e intervenciones de los jugadores.

Cuadro 5. Destrezas psicológicas que debe poseer entrenador para mejorar sus intervenciones en los periodos de participación activa de intensidad menor

Control emocional.

Toma de decisiones

Mejora de la comunicación verbal

Mejora de la comunicación no verbal

Atención-concentración

Autoconfianza

Cuadro 6. Actuaciones de los entrenadores en los periodos de participación activa de intensidad menor

\begin{tabular}{|l|}
\hline Observar al propio equipo y al equipo contrario \\
Introducir cambios tácticos y estratégicos \\
Comunicación verbal con auxiliares \\
Mostrar una actitud de confianza y control \\
Pausar y calmar al equipo o activar estimular \\
Realizar adecuadamente la solicitud temporal de los tiempos \\
Muertos \\
Sustituir a los jugadores adecuados \\
Cuidar la comunicación no verbal \\
Interpretar adecuadamente los diferentes momentos partido \\
\hline
\end{tabular}

\section{Intervenciones en periodos de participación activa de intensidad menor}

Los periodos de participación activa de intensidad menor son muy apropiados para interpretar el ritmo de los partidos. Se pueden utilizar para introducir los cambios mas adecuados. Con los tiempos muertos se pueden introducir las modificaciones tácticas que el entrenado estime oportunas. Nuestra experiencia nos revela que para sacar el máximo aprovechamiento a estos trascendentales momentos de la dinámica de un partido se necesita un buen entrenamiento mental del entrenador.

También debemos destacar que es en estos momentos cuando los entrenadores deben tomar decisiones de los posibles cambios o sustituciones de jugadores. Esta intervención, generalmente táctica, debe ir acompañada de la correcta interpretación del periodo inmediatamente anterior de participación activa intensa y acompańada de la consiguiente información individual táctica.

\section{Actuaciones y participación de los jugadores en los periodos de participación activa de intensidad menor}

En los periodos de participación activa de intensidad menor los jugadores suelen realizar intervenciones que surgen por la iniciativa propia. Otras actuaciones de los jugadores suelen responder a las propuestas realizadas por los entrenadores,

Una las fases más interesantes de los periodos de participación activa de intensidad menor son las de cambio de complejo táctico. En estas fases se suelen producir contactos que facilitan la comunicación intergrupal. También, son momentos en que los jugadores tratan conseguir adecuados estados de activación.

Durante ellos se ocasionan conductas grupales que pretenden mantener un buen ambiente de juego En un trabajo de Díaz (1984) observó que durante partidos de voleibol femenino algunas jugadoras llegaban a realizar mas de trescientas acciones no relacionadas con acciones técnicas o tácticas. Las conductas como; levantar los brazos, gritar, golpear la mano de otra compañera, patear el suelo, sonreír, felicita o saltar eran diferentes según el equipo hubiera conseguido el punto o le hubieran fallado. En el mencionado trabajo se observó que esos gestos se podían dirigir hacia la misma jugadora o hacia su grupo. Estos comportamientos siempre se realizaban en los periodos de participación activa de intensidad menor. Según Díaz (1984) con estos comportamientos en estas fases del juego se consigue una adaptación psicológica al momento de juego individual y grupal.

También, los jugadores aprovechan estos momentos de participación menos activa para ayudarse a identificar estímulos discriminatorios relevantes del juego. En algunos casos estos estímulos relevantes ayudan a realizar tareas de anticipación. 
Los jugadores de los equipos mas experimentados y entrenados psicológicamente aprovechan estos momentos para realizar una correcta comunicación de intercambio de información sobre los rivales y sus estrategias de juego. Igualmente son muy adecuados estos subperiodos para realizar intervenciones sobre la necesidad de mantener adecuadamente los niveles de atención o concentración necesaria para las fases de juego de intensa actividad. Los entrenadores deben promover conductas apropiadas en estos periodos que ayuden a conseguir los estados psicológicos referidos

En estos subperiodos de de cambio de complejo dentro de los periodos de actividad de intensidad menor, los jugadores y entrenadores tienen diferentes comportamientos e intervenciones intragrupales distintas según el resultado de la anterior actividad.

Una secuencia de este subperiodo se desarrolla de la siguiente manera. Cuando el resultado ha sido positivo, los jugadores suelen intercambiar refuerzos sociales y verbales intentando estimularse y mantener un elevado grado de activación. Seguidamente los jugadores recuperan sus posiciones en el campo. En caso de los jugadores que se encuentran en el complejo táctico KII, suelen comunicarse entre ellos, mientras que los jugadores en defensa se comunican menos. Esta situación de intercambio de información se debe a la proximidad física. Por lo general el entrenador interviene principalmente dando información al jugador que saca y seguidamente sobre los jugadores delanteros aconsejando sobre el bloqueo.

En el caso de los jugadores que se encuentran en el complejo táctico KI, se comunican menos, pues su trabajo táctico es mas intenso. Por lo general, suele haber una mayor intervención del entrenador.

En el caso contrario, estos es, cuando el resultado ha sido negativo, los jugadores intercambian menos refuerzos sociales. En la mayoría de las ocasiones el, intercambio se limita a algún toque de manos individualizado y algún jugador que estimula verbalmente al colectivo. Precisamente es en estos momentos en los que el entrenamiento psicológico sería más efectivo para ser capaz de recuperar y olvidar el error. Seguidamente los jugadores recuperan su posición en el campo.

\section{Periodos de pausa}

Los periodos de pausa en voleibol son:

- Tiempos muertos solicitados por los entrenadores

- Tiempos técnicos

- Intervalos entre sets

Como señala (Buceta (2004) La intervención psicológica durante los periodos de pausa tiene como objetivo que los deportistas aprovechen estos momentos en su beneficio, en lugar de desaprovecharlos o que incluso perjudiquen su funciona- miento. Este posicionamiento inicial debe ser el referente del trabajo del entrenador durante los periodos de pausa.

Los tiempos muertos y técnicos con una duración de 30 segundos son cuatro durante cada set, excepto en el quinto set en el que solamente habrá uno. En resumen, y siempre que se soliciten los dos a los que tienen derecho reglamentario el entrenador, cada entrenador podrá disponer de seis momentos para realizar actuaciones e intervenciones sobre jugadores. Existen algunas diferencias fundamentales entre tiempos técnicos y tiempos muertos. Los tiempos técnicos siempre suceden en los mismos momentos, cuando uno de los equipos llega a los tantos 8 y 16 y tienen una duración de 60 segundos. Son tiempos obligatorios. Los tiempos muertos son solicitados por el entrenador cuando lo consideré oportuno. Estos tiempos muertos también presentan una diferencia que se debe tener presente. Cada entrenador solicita dos tiempos que pueden ser utilizados por ambos entrenadores, aunque, posiblemente la intervención sea distinta en cada uno de ellos. Es importante tener prevista esta circunstancia.

Actuaciones de los entrenadores en los periodos de pausa.

Aunque la situación referida en el apartado anterior puede y debe condicionar la actuación de los entrenadores, sobre todo para las indicaciones o informaciones técnicas, en general, el entrenador debe mantener una adecuada preparación psicológica personal para abordar estos periodos de pausa de forma que resulten eficaces y ayuden a mejorar el rendimiento individual y grupal.

Como norma habitual el entrenador se debe presentar ante sus jugadores durante el periodo de pausa calmado pero involucrado en el juego. En todo momento deberá dominar y controlar la situación y procurará una comunicación directa y precisa. Como en muchas ocasiones, y como hemos comentado en otros apartados de este trabajo, los periodos de pausa ocurrirán o se solicitarán en momentos de un alto grado de activación e incluso de ansiedad competitiva. El entrenador deberá poseer un alto control emocional para poder controlar los estados de ansiedad de sus jugadores.

Cuadro 7. Habilidades psicológicas de los entrenadores en los periodos de pausa.

Comunicación directa
Calmado pero involucrado
Control de las emociones
Dominio de la situación
Control de niveles de activación
Toma de decisiones

Los periodos de pausa deben utilizarse para conseguir una adecuada activación psicológica. Los jugadores pueden acudir a los periodos de pausa en ocasiones sobre activados y en 
otros momentos con grados de activación bajos. El grado de activación dependerá de las diferentes circunstancias del juego, del partido, de la competición y también de variables de personalidad de los jugadores. El entrenador deberá interpretar adecuadamente la activación que presenta su equipo e intervenir sobre ella., Nuestra experiencia y las observaciones y opiniones recogidas entre el colectivo de entrenador, nos indican que, por lo general, los jugadores suelen llegar con estados de activación excesiva a los periodos de pausa.

Puede haber diferencias de grados de activación cuando los tiempos muertos son solicitados por el entrenador rival y cuando son solicitados por el entrenador propio. También puede haber diferencias en las pausas por los tiempos técnicos.

Como regla general podemos asegurar que no hay ningún periodo de pausa igual. Todos los tiempos de pausa tienen características distintas y requieren actuaciones distintas.

No obstante, proponemos que se deberían implantar rutinas en estos periodos de pausa para conseguir un adecuado funcionamiento psicológico.

Los jugadores suelen llegar a los periodos de pausa con una activación excesiva. En este estado los jugadores no suelen poder atender adecuadamente a las informaciones e intervenciones del entrenador. Balaguer (1993) manifiesta que un estado de activación excesiva produce falta de concentración y conductas de precipitación en el momento de tener que tomar decisiones.

El empleo de rutinas se ha revelado como una práctica útil a la hora de establecer pautas de comportamiento óptimas tanto en la pre-competición como durante la competición y post-competición en distintas modalidades deportivas (Díaz, 2003; Garcés de los Fayos, Pelegrin y González, 2009; Olmedilla, Jara y Andreu, 2009. Almeida y Olmedilla, 2009; Palmi, 1991; Jaenes y Caracuel, 2005). Las rutinas permitirán a los jugadores elegir aquellos aspectos que les son más útiles para adecuar su nivel de activación. El nivel de activación adecuado le posibilitará una mejor atención a las demandas del entrenador. Por lo tanto, el establecimiento de alguna rutina durante los periodos de pausa potenciarían los requerimientos psicológicos necesarios para aprovechar mejor estos periodos.

\section{Rutinas en los periodos de pausa}

Durante esos segundos que duran los tiempos de pausa, y como hemos mantenido anteriormente, los jugadores deben aprovecharlos en mejorar su funcionamiento psicológico.

Por regla general se deberían instaurar las siguientes pautas de actuación. Los jugadores deberían acudir con presteza a la zona de banquillo y aprovechar todo el tiempo. En primer lugar tratar de hidratarse adecuadamente y acondicionar su vestuario. Una buena rutina es que se sienten y haya unos segundos de pausa y relajación. Que sea una sola persona la que hable y hacerlo sin precipitarse, con frases completas y claras. (Decir lo que se quiere decir). Apartar la atención de las últimas jugadas negativas. Reforzar las mejores jugadas, decisiones y actuaciones anteriores. Establecer las prioridades sobre las actuaciones inmediatas a la vuelta al campo. El entrenador debe centrarse en las acciones concretas que quiere que haga cada uno de los jugadores.

Como hemos dicho anteriormente debemos tener previsto que el periodo de pausa puede ser distinto según lo pida un entrenador u otro o sea un tiempo técnico. Si durante el periodo de pausa las cosas están saliendo según lo previsto y van considerablemente bien, habrá que reafirmarse en seguir la estrategia de juego que se esta llevando a cabo, al tiempo que se trasmiten ideas y sentimientos de autoafirmación y confianza. Por el contrario, si en el tiempo de pausa las cosas van mal habrá que decidir si es el momento de introducir cambios o proponer alternativas personales y jugadas ensayadas.

Cuadro 8. Modelos de rutinas durante los periodos de pausa

Acudir con rapidez a la zona del "banquillo"
Hidratarse adecuadamente.
Acondicionar vestuario
Momentos de calma y relajación
Hablar solamente una persona
Hablar sin precipitarse: frases completas y claras (decir lo que
se quiere decir)
Apartar la atención de las últimas jugadas negativas
Reforzar las mejores jugadas, actuaciones y decisiones
Establecer prioridades: actuaciones inmediatas a la vuelta al
juego
Centrarse en las acciones concretas que quiere que cada uno
haga

Es muy importante tener preparadas las rutinas de los tiempos de pausa y repetirlas invariablemente. Tener presente que hacer en función de que las cosas vayan bien y que alternativas tenemos cuando las cosas vayan mal.

\section{Fase postcompetitiva}

"El periodo inmediato de finalización de los periodos competitivos es de una extraordinaria importancia ya que en este periodo, en ocasiones, se suelen poner las bases del siguiente periodo competitivo". (Buceta, 1998)

Según diferentes autores:( Buceta, 1998; García Mas 1997; Dosil, 2002) es recomendable que el entrenador no realice la intervención de la fase post-competitiva inmediatamente después del partido, sino que aplace sus comentarios para el día siguiente o para el comienzo de la siguiente sesión de entrenamiento.

Nada más finalizar el partido la capacidad emocional de los jugadores, e incluso del entrenador no entrenado psicológicamente, puede estar afectada según haya sido su experien- 
cia en el partido: enfado, alegría, tristeza, nervios, crispación, cansancio. Si el entrenador se encuentra afectado emocionalmente puede realizar la interpretación y el análisis erróneos de lo sucedido durante el partido

En la siguiente sesión de entrenamiento, el entrenador podrá utilizar la experiencia del partido anterior para mejorar los objetivos de la sesión. Esta actuación es muy adecuada para el aumento de la motivación y de la atención de los jugadores ya que suelen estar muy receptivos. Tanto a nivel grupal como individual las actuaciones e intervenciones post-parti- do suelen ayudar a la mejora individual y de grupo.

\section{Actuaciones del entrenado en la fase postcompetitiva}

El control emocional del entrenador debe presidir las actuaciones post-partido. Los entrenadores psicológicamente orientados deberán realizar sus intervenciones en las fases post-partido distinguiendo las cuatro situaciones que a juicio de Martens (1989) y Dosil (2004) se pueden dar después de una competición.

Cuadro 9. Intervenciones del entrenador post-partido según las situaciones del resultado

\begin{tabular}{ll}
\hline El equipo sale derrotado pero ha cumplido una buena actuación & $\begin{array}{l}\text { El entrenador debe enfatizar sobre el rendimiento individual y grupal y } \\
\text { sobre el esfuerzo y el trabajo realizado }\end{array}$ \\
\hline El equipo sale derrotado después de una mala actuación. & $\begin{array}{l}\text { El entrenador basará su intervención en la insistencia en la mejora de la } \\
\text { calidad del trabajo en los aspectos, físicos, técnicos, tácticos y psicológicos }\end{array}$ \\
\hline El equipo ha ganado después de una buena actuación & $\begin{array}{l}\text { Destacar el esfuerzo individual y colectivo basado en la calidad del entre- } \\
\text { namiento, insistiendo en el rendimiento constante durante los partidos }\end{array}$ \\
\hline El equipo ha ganado pero ha realizado una mala actuación. & $\begin{array}{l}\text { El entrenador dedicará su intervención a enfatizar sobre los aspectos que } \\
\text { habrá que mejorar en el siguiente partido }\end{array}$ \\
\hline
\end{tabular}

Es muy conveniente y recomendable ayudarse, para las intervenciones post-partido, de registros objetivos sobre el rendimiento y comportamiento de los jugadores. El video, las plantillas de registros y otros medios que ayuden a cuantificar acciones eliminarán las interpretaciones erróneas y las impresiones personales del entrenador. El entrenador y los jugadores podrá analizar mejor lo sucedido y la intervención será de mayor calidad

En definitiva, al realizar las intervenciones post-partido el entrenador deberá mostrar una actitud controlada, positiva y constructiva en la que deberá destacar los aspectos y situaciones positivas y dando alternativas y proponiendo soluciones grupales e individuales.

\section{Conclusiones y reflexión}

El voleibol es un deporte muy secuenciado y cíclico con diferentes momentos de participación de los jugadores en el juego y con diferentes momentos de parada y/o de espera. Los jugadores además no tienen una participación homogénea en el juego. Nos encontramos con jugadores más protagonistas y otros que tienen un protagonismo secundario. Los entrenadores cuentan con diversas formas de intervenir en el juego de su equipo mediante actuaciones en las distintas fases de un partido. La pormenorización y análisis de las características que presentan las distintas fases, periodos y subperiodos que hemos propuesto en este trabajo ayudarán a los entrenadores a ajustar y adecuar sus intervenciones teniendo en cuenta las particularidades de cada fase competitiva. Aunque hemos recogido también las actuaciones de los jugadores en dichas fases de juego, resultaría interesante ampliar este trabajo con actuaciones concretas para los jugadores, que posiblemente les permitiría una mejora de actuación ínter e intragrupal con una mayor implicación personal en los aspectos motivaciones y de creación de ambiente de juego.

Por último, hemos destacado en diferentes momentos del trabajo, la trascendencia de la formación psicológica de los entrenadores.

Como mantienen numerosos psicólogos del deporte (Cantón, 2009; Cruz, 1997, Dosil, 2002; Guillen 2003; Buceta, 1999), la formación en psicología de los entrenadores y su entrenamiento psicológico tiene una extraordinaria importancia en la actuación de los entrenadores tanto en la competición como en los entrenamientos. En este trabajo hemos destacado su importancia en la faceta de la dirección del equipo durante los partidos.

Este entrenamiento personal no solo proporcionará a los entrenadores un mayor bienestar psicológico, sino que además le servirá para mejorar las estrategias y habilidades psicológicas de comunicación, de control emocional, de toma de decisiones, de credibilidad y de mejora de la atención-concentración. También le será de una gran utilidad y le apoyará de forma eficaz en las funciones principales de los entrenadores de equipos, la dirección de equipo durante la competición

Para terminar, insistir en que este tipo de trabajos en los que se estudian y analizan las dinámicas de deportes concretos. En los que se profundiza sobre peculiaridades concretas en las que la faceta mental puede tener mayor protagonismo, puede ser una de las aportaciones de la psicología del deporte al trabajo aplicado. En este trabajo hemos recogido aportaciones concretas con las que los psicólogos del deporte podemos favorecer el trabajo de técnicos y entrenadores de voleibol en la facetas de trabajo en dirección del equipo durante los partidos. 


\section{Referencias}

Almeida, P. y Olmedilla, A. (2009) “Bed on Tour": Impacto de las rutinas extradeportivas en un surfista profesional. En J. Díaz, I. Díaz y J. Dosil (eds.) La Psicología del deporte en Iberoamérica pp 149-150. Torrelavega. SIPD.

Alonso, C.; Boixados, M. y Cruz, J. (1995) Asesoramiento a entrenadores de baloncesto: efectos en la motivación deportiva de los jugadores. Revista de Psicología del deporte 7-8, 135-146.

Balaguer, I. (1993) Entrenamiento psicológico, en M. Crespo (coord...) Tenis, I, pp 273-292. España COI.

Balaguer, I. (2007) Clima motivacional, calidad de la implicación y bienestar psicológico: una propuesta de intervención en equipos deportivos. En A. Blanco y J. Rodríguez Marín (Eds.). Intervención Psicosocial pp 135-162 Madrid: Pearson-Prentice Hall.

Beal, D. (1989) Sistema y tácticas básicas de equipo. Manual para entrenadores de la FIVB. Nivel I. C. $5^{\circ}$ pp170-188 Laussane. FIVB.

Boixados, M. y Cruz, J. (1999): Intervención conductual en entrenadores de futbolistas alevines. En F, Guillen (Ed.) La psicología del deporte en España al final del milenio. (pp 423-431). Las Palmas de gran canaria: Servicio de Publicaciones de la Universidad de Las Palmas de Gran Canaria

Buceta, J. Ma (1998) Psicología del entrenamiento deportivo. Madrid. Dykinson.

Buceta, J. Ma (1999) Psicología de los entrenadores deportivos. Apuntes del master de psicología de la actividad física y del deporte. Madrid, UNED. CSD FUyE.

Buceta, J. Ma. (2004): Estrategias psicológicas para entrenadores de deportistas jóvenes. Madrid: Dykinson.

Cantón, E. (2006) El ejercicio profesional en psicología del deporte en la actividad física y el deporte. En E.J. Garcés de los Fayos, P. Jara y A. Olmedilla (coord.) Psicología y deporte. Murcia. Diego Marín.

Cantón, E. (2009) La profesión del psicólogo del deporte. En J. Díaz, I. Díaz y J.Dosil (eds.): La psicología del deporte en Iberoamérica (pp161). Torrelavega. Cantabria): SIPD.

Cloes, M.; Delhaes, J.P. y Pieron, M. (1993). Analyse des comportments d'entraineurs de volleyball pedant des rencontres officielles. Sport, 141, $16-25$

Cruz, J. (1997) Psicología del deporte. Madrid. Síntesis.

Cunha, P. (1998): A Intervençao do treinador durante o tempo morto. Treino Desportivo, 1-2. 33-38.

Díaz, J (1984): Observación y registro de gestos paradeportivos durante un encuentro de voleibol femenino de la primera división española. Actas del coloquio internacional de psicología del deporte. Madrid. CSD. Vb. Separata técnica No 19-20-21-22.

Díaz, J. y Rodríguez, G. (2005): Intervención psicológica mediante rutinas de atención y concentración en un equipo de voleibol para mejorar la efectividad colectiva del saque. Cuadernos de Psicología del deporte, 5 (1-2) 219-230.

Díaz, J. (2003) Psicología de los Bolos. Santander. Federación Cantabra de Bolos.

Díaz, P. (1992) La dirección de equipo. En Voleibol Español 289-316. Madrid. COI.

Dosil, J. (2002) Preparación psicológica de las competiciones en J. Dosil (ed.). Psicología y rendimiento deportivo. Ourense. Gersam.

Dosil, J. (2004) Psicología de la actividad física y del deporte. Madrid. Mc Graw hill.

Garcés de los Fayos, E.; Pelegrín, A. y González, J. (2009) Entrenamiento psicológico aplicado en diferentes modalidades deportivas. En J. Díaz, I. Díaz y J. Dosil (eds.) La Psicología del deporte en Iberoamérica pp 150 154. Torrelavega. SIPD.

García Más, A. (1997) Análisis psicológico del equipo deportivo. Las bases del entrenamiento deportivo, en J. Cruz (coord.), Psicología del deporte 193-214. Madrid: Síntesis.

Giménez, F. J. (2003) La formación del entrenador en la iniciación al baloncesto. Sevilla. Wanceulen.

Guillen, F. (2003): Psicopedagogía de la actividad física y el deporte. ArmeniaColombia. Kinesis.

Herrera, G.; Ramos, J. L. y Mirella, J. (1989): Dirección de Equipo en el alto rendimiento. Cuadernos para entrenadores de voleibol. Bilbao Federación Vasca de Voleibol.

Hotz, A. (1999): Corregir apenas o estrictamente lo necessario, variar o mais possivel. Trenio. Desportivo, 2, 6 22-36.

Jaenes, J.C y Caracuel, J.C. (2005). Maratón. Preparación psicológica para el entrenamiento y la competición. Córdoba. Almuzara.

Martens, R. (1989) El entrenador. Madrid. Hispano Europea.

Mesquita, I.; Gisela, R.; Rosado, A.; Moreno, P. (2005) Análisis de contenido de la intervención del entrenador de voleibol en la reunión de preparación para la competición. Estudio comparativo de entrenadores de equipos senior masculinos y femeninos. Barcelona Apunts: Educación física y deportes. 81, 61-66.

Moreno, P.; Villar del, F.; Santos, J. A. (2005). La comunicación del entrenador de voleibol durante la dirección de equipo en competición. Madrid. RFEVb.

Moreno, P. Santos, J. A Ramos, L.; Sanz, D.; Fuentes, P. (2002) Aplicación de un sistema de codificación para el análisis de contenido de la conducta verbal del entrenador de voleibol. Motricidad; Revista de ciencias de la Actividad Física y del Deporte. 9, 119-140.

Olmedilla, A.; Jara, P.; Andréu, M.D (2009): Momentos críticos y Toma de decisiones del Psicólogo en el entrenamiento psicológico de deportistas. . En J. Díaz, I. Díaz y J. Dosil (eds.) La Psicología del deporte en Iberoamérica p. 55. Torrelavega. SIPD.

Palmi, J. (1991) Entrenamiento psicológico para la competición. En J. Riera y J. Cruz (eds) Psicología del deporte. Aplicaciones y Perspectivas. Barcelona. Martínez Roca.

Romero, M. P. (2008) Sensibilización, Formación e Intervención psicológica en fútbol base. En J. Díaz, I. Díaz y J. Dosil (Eds) La Psicología del deporte en Iberoamérica (pp.50-53) Torrelavega. SIPD.

Santos. J. A. (1992): La táctica colectiva. En voleibol español 133-165. Madrid. COI.

Susan, A y Mihaly, C. (2002): Fluir en el deporte. Barcelona. Paidolibro.

Ureña, A. (1992).Planificación. En Voleibol español 249-279. Madrid-COI.

Velasco, J (2009) La Formación del Equipo Nacional Español: bases y principios para la creación de un modelo propio. Actas VIII Congreso Internacional sobre entrenamiento en Voleibol. Valladolid. RFEVb. 